
allemande

50-1 | 2018

Regards franco-allemands sur la justice dans la construction européenne | Les élections législatives de 2017 en Allemagne et en Autriche

\title{
Brigitte Sauzay, une belle figure des relations franco-allemandes
}

\section{Martine Sauzay}

\section{(2) OpenEdition \\ Journals}

Édition électronique

URL : https://journals.openedition.org/allemagne/675

DOI : 10.4000/allemagne.675

ISSN : 2605-7913

Éditeur

Société d'études allemandes

Édition imprimée

Date de publication : 30 juin 2018

Pagination : 199-205

ISSN : 0035-0974

Référence électronique

Martine Sauzay, « Brigitte Sauzay, une belle figure des relations franco-allemandes », Revue d'Allemagne et des pays de langue allemande [En ligne], 50-1 | 2018, mis en ligne le 30 juin 2019, consulté le 02 juin 2022. URL : http://journals.openedition.org/allemagne/675 ; DOI : https://doi.org/ 10.4000/allemagne.675 


\section{Brigitte Sauzay, une belle figure des relations franco-allemandes}

- Martine Sauzay

Le 14 octobre 2017 avait lieu dans les locaux du Centre interdisciplinaire d'études et de recherches sur l'Allemagne (CIERA) à Paris une cérémonie de remise d'un ouvrage de mélanges en l'honneur d'Anne-Marie Saint-Gille qui fut professeur de civilisation allemande à l'Université Lyon 2. Une table ronde réunissant des germanistes a été l'occasion d'échanges sur la thématique "conflit et coopération" au centre de l'ouvrage d'hommage paru chez Peter Lang et édité par Jean-Michel Pouget. En ouverture de cette table ronde, Martine Sauzay-Mandereau, professeur de lettres classiques et membre du comité d'administration de la fondation Genshagen, a évoqué le souvenir de sa sour Brigitte Sauzay, grande figure de la coopération franco-allemande jusqu'à sa disparition prématurée en 2003. Voici le texte de ce témoignage émouvant.

Jean-Michel Pouget Université Paris-Nanterre

Je suis l'intrus dans la série devant vous tous germanistes confirmés, je suis seule ici à ne pas parler l'allemand et pourtant ma proximité avec une personne dont la carrière a marqué les relations franco-allemandes m’amène à penser que mon témoignage peut être intéressant. C'est du moins l'avis de Jean-Michel Pouget à l'occasion de cet hommage rendu à Anne-Marie Saint-Gille qui a axé sa réflexion sur la construction de la paix en Europe à travers l'étude des relations franco-allemandes. Il y a, à mon sens, une certaine similitude, au moins des ressemblances, entre l'ambition de Brigitte Sauzay et celle d'Anne-Marie Saint-Gille: mon évocation vous permettra peut-être de le constater.

Je rappelle que Brigitte Sauzay fut d'abord interprète de conférence en free-lance, c'est-à-dire à titre libéral, avant de devenir peu à peu l'interprète officielle du Quai d'Orsay pour l'allemand, jusqu'au jour où elle est passée de la traduction au conseil auprès des politiques. Ses deux livres, Le Vertige allemand (1985) puis surtout Retour à Berlin (1997), l'ont fait connaître d'un public plus large en France comme 
en Allemagne et ce d'autant plus qu'elle avait fondé avec le professeur et historien Rudolf von Thadden au château de Genshagen, près de Berlin, un institut pour les relations franco-allemandes qui, dès les années 1990, permit aux intellectuels de l'Est et de l'Ouest, français et allemands, d'aborder ensemble toutes sortes de sujets politiques et culturels. Le rayonnement de cette institution, l'appui de Rudolf von Thadden, son mentor dans l'Université allemande, sa connaissance des questions politiques acquise au sein du Quai d'Orsay et surtout lors des sommets européens auprès des chefs d'État, le soutien enfin du président François Mitterrand, tout ceci a finalement attiré l'attention de Gerhard Schröder, chef de file des sociaux-démocrates allemands, qui lui a proposé, s'il devenait chancelier, d'être auprès de lui à Berlin à la chancellerie toute neuve, sa conseillère particulière pour les relations franco-allemandes.

Mais beaucoup d'entre vous connaissent ce parcours et la question qui vous intéresse est peut-être la suivante: comment devient-on Brigitte Sauzay, et comment s'est-elle située parmi les passeurs de culture qui ont marqué l'histoire des relations franco-allemandes? Comme toujours, c'est la conjonction d'une personne et d'une époque.

Si nous jouons au jeu des poupées russes, nous dirons qu'une personne c'est l'imbrication de l'inné et de l'acquis, le fruit de la loterie génétique dans un milieu donné. Brigitte est née en 1947 dans un milieu de moyenne bourgeoisie, marqué par la présence d'officiers de différentes armes, un milieu durement éprouvé par les guerres successives, pour qui l'Allemagne c'est l'ennemi et même plus: ma mère avait coutume de nous raconter qu'enfant, dans les années 1930, son père, médecin colonial, l'avait emmenée à Strasbourg à proximité du pont de Kehl en lui disant: «Tu vois, là-bas, c'est l'Allemagne» et qu'elle avait alors pensé entrevoir l'Empire du Mal. On racontait à la maison la mort héroïque de notre oncle aviateur basé en Angleterre alors qu'il bombardait la Ruhr, et bien d'autres histoires. Quand, des années plus tard, Brigitte, au cours de ses études, amena des amis allemands à la maison et que deux jeunes gens s'inclinèrent devant ma mère en claquant des talons «Bismarck, Schlieffen", je crus que ma mère allait s'évanouir.

En même temps mes parents vivaient, l'un comme l'autre, dans l'admiration du général de Gaulle, l'Homme du 18 juin, et étaient parfaitement prêts à le suivre dans une politique de réconciliation qui leur paraissait d'abord hautement souhaitable pour éviter de nouvelles guerres. L'allemand, c'était donc la langue de l'ennemi, un ennemi qu'il faut connaître pour le combattre, mais aussi pour tenter une transsubstantiation d'ennemi en ami. Si on ajoute un zeste de christianisme social dont étaient porteuses les familles paternelle et maternelle, cette alchimie est compréhensible. Plus généralement l'empreinte chrétienne prédisposait à cette attitude.

Et puis l'allemand c'était difficile, donc attirant et jouissant d'un prestige intellectuel. L'été, invariablement passé à la campagne dans les maisons de famille où malgré de nombreux cousins on s'ennuyait un peu, on tombait sur des grammaires allemandes, des volumes de poésie bilingue. Brigitte a pu y être sensible (je me rappelle avoir lu ainsi les dix tomes du Mémorial de Sainte-Hélène de Las Cases, la poésie de Vigny et Musset... mais c'est une autre histoire). Mon père, en Européen convaincu et en avance sur son temps avait décidé d'envoyer ses enfants un an dans un collège anglais. 
À son retour Brigitte se vit évidemment proposer de passer à l'étude intensive... de l'allemand. L'essentiel, pour mon père, c'était de ne pas être «médiocrrre»!

Pour bien décrire ce milieu social il faudrait ajouter qu'on y avait la tête politique: nous «aimions la France» dont nous nous faisions «une certaine idée», pleinement inscrits dans une tradition à la fois laïque et catholique qui remonte à Michelet et qui fait de la France plus qu'un concept, une personne: mes parents n'auraient manqué ni le défilé du 14 juillet ni une émission politique, en un temps où elles étaient peu nombreuses mais très suivies.

Je restitue ici un climat familial, un ensemble de données mais rien de décisif: il faut aussi un tempérament et un événement déclencheur. Le tempérament d'abord: Brigitte quoique de santé un peu fragile avait une grande énergie, un grand amour de la vie, le goût de plaire et une ambition personnelle: je l'entends encore, elle devait avoir 15 ou 16 ans, «Je serai la grandesse Brigitesse, Sauzaytesse poétesse» ou plus jeune encore, parlant du journal, «On lit Le Monde, mais pourquoi on n'est pas dedans?»

Quant à l'événement, le voici: au cours de sa licence Brigitte partit pour un an à Fribourg-en-Brisgau et là se produisit un phénomène particulier : dans cette ville, il est vrai séduisante, elle tomba amoureuse de l'Allemagne et noua des amitiés de toute une vie - et je dirai qu'à partir de là elle ne cessa jamais d'avoir avec l'Allemagne, et jusqu'à sa mort en 2003, une relation passionnée.

Mais l'ambition ne suffit pas: pour marquer, comme elle l'a fait je crois, l'histoire des relations franco-allemandes, il faut aussi être porteur d'une vision et être d'un caractère décidé. Et Brigitte avait une vision, où je crois que le réalisme se mariait assez heureusement avec l'idéalisme. L'article de Reiner Markowitz, dans l'ouvrage dédié à Anne-Marie Saint-Gille ${ }^{(1)}$, nous le rappelle excellemment et m’a frappée: le franco-allemand a pu paraître à la fin des années 1960, dans les années 1970 ou 1980, quelque chose d'un peu figé, une sorte de dogme aux mains d'un très petit nombre d'intellectuels et de hauts-fonctionnaires. Brigitte a découvert ce milieu très jeune: née en 1947, elle a connu son premier dîner à l'Élysée, introduite par son professeur à l'école d'interprètes, sous le président Georges Pompidou, donc avant 1974 (elle n'a pas 25 ans); et l'intimité des puissants, parfaitement acquise sous la présidence de Valéry Giscard d'Estaing, alors qu'elle est de tous les sommets Giscard-Schmidt, lui révèle sans doute une part du dessous des cartes, empêche sa vision d'être trop irénique, trop idéaliste. Elle observe, elle écoute, tout en étant tenue au secret professionnel et je me rappelle fort bien que la famille tout entière a été soumise à une enquête de police approfondie avant que Brigitte ne devienne pour l'allemand l'interprète attitrée des réunions au sommet. Elle a d'ailleurs eu du mal à se faire une place dans ce qu'on appelait d'un bloc «le franco-allemand», elle a suscité une jalousie dont elle a évidemment souffert.

Pour y faire face, elle s'est montrée combative, très professionnelle dans son travail d'interprète et observatrice attentive des phénomènes de cour. Mais elle a aussi

1 Reiner Markowitz, «Le plan Schuman et le traité de l'Élysée: deux mythes fondateurs du rapprochement franco-allemand», in: Jean-Michel Pouget (éd.), Conflit et coopération. France-Allemagne XIX ${ }^{e}-X X^{e}$ siècle. Mélanges en l'honneur d'Anne-Marie Saint-Gille, Berne, Peter Lang, 2017, p. 259-278. 
réagi par l'étude: ne voulant pas enseigner (un métier où on ne peut pas "réussir», me disait-elle pour me décourager!), elle a pourtant beaucoup appris. Sa situation durant ces années 1970 puis 1980 est assez particulière et formatrice: à la faveur des conférences, elle découvre des milieux dont elle ignorait tout, pas seulement le milieu des interprètes de conférences, milieu assez dur, où la rivalité est grande, où l'on trouve de fortes personnalités issues de familles diversifiées, multilingues, mais aussi le milieu chaque fois neuf, original, des différents métiers qui recrutent ponctuellement des interprètes et enfin celui des politiques - et c'est celui-là qui l'intéresse le plus.

Remarquée par le président Giscard d'Estaing, elle ambitionne de ne plus seulement traduire mais d'avoir de l'influence. Ses préoccupations à l'époque tournent autour de la comparaison entre les deux sociétés, allemande et française, et elle s'interroge sur l'origine de ces différences.

L'élection du président François Mitterrand lui fait craindre de perdre sa position: ce sera tout le contraire. Nommée au service d'interprétation du Quai d'Orsay, désormais titulaire aux Affaires étrangères, elle suit des cours à l'École des hautes études en sciences sociales (EHESS) pour approfondir ses connaissances, et c'est alors qu'elle rencontre celui qui va lui ouvrir la porte de l'Université allemande, Rudolf von Thadden. Cette rencontre a certainement facilité ses progrès et dans la connaissance de l'Histoire et dans la reconnaissance par le milieu universitaire.

Rudolf von Thadden n'était pas seulement un historien reconnu, il avait des responsabilités dans l'Église évangélique allemande, appartenait à l'aristocratie prussienne, celle qui avait souffert du nazisme. C'est avec lui qu'elle a fondé, tout de suite après la chute du Mur, qu'elle vécut comme un événement personnel, l’institut Berlin-Brandebourg pour les relations franco-allemandes à Genshagen près de Berlin. C'était une sorte de mariage entre la politique et l'Université dans un de ces châteaux déchus dont l'Allemagne de l'Est finissante cherchait alors à se débarrasser, et les colloques à dominante politique ou économique, sociale et culturelle ont été, je crois, d'emblée et dans l'ensemble, une réussite. On venait en tant qu'universitaire ou membre du gouvernement faire une communication mais aussi dîner, prendre un verre, poursuivre la discussion le soir avec le sentiment d'être reçu au château, dans cette campagne du Brandebourg un peu loin de tout et un peu sévère, dans une atmosphère très allemande, d'une grande simplicité, où tel ministre poussait son plateau entre deux personnalités du monde économique ou religieux. Je crois que Brigitte ambitionnait de reconstituer l'atmosphère des salons du XVIII ${ }^{\mathrm{e}}$ siècle pour la liberté d'esprit, la capacité à brasser tous les sujets, à faire se rencontrer des gens de formations diverses dans un beau cadre - elle avait un peu en tête Madame de Staël - évidemment sur un mode plus démocratique, plus moderne et moins argenté!

Vous connaissez la formule du Canadien Mc Luhan: le media (ou le medium), c'est le message! Quel était ce message?

En dehors des dures réalités des rapports étatiques, de la conscience qu'il s'agissait d'abord d'aider à l'équilibre d'intérêts contraires en tenant compte de la puissance allemande (dont on se souvient qu'elle inquiète particulièrement en ces temps de réunification), il y avait une part évidente d'humanisme, d'idéalisme contenue dans l'affirmation suivante: «Nous sommes une famille! France et Allemagne sont 
intimement complémentaires». J'ai retrouvé dans le bel article de Jean-Michel Pouget consacré à Paul Distelbarth ${ }^{(2)}$ cette atmosphère de foi en l'Europe, dispensatrice de paix, foi ancrée tout autant dans le romantisme français, et qui à travers Hugo remonte à Condorcet, à son esquisse d'un tableau historique des progrès de l'esprit humain, chapitre $\mathrm{X}$.

Je crois que le succès de Brigitte s'explique en partie par l'affirmation de sa foi en l'Europe alors même qu'elle était parfaitement au contact rugueux des rivalités inhérentes au choc des intérêts politiques et économiques. Je voudrais me souvenir aujourd'hui, même si on a peine à l'imaginer, de l'émotion avec laquelle ce matin de janvier au début du siècle, nous sommes allées en tremblant presque et je n'exagère pas, Brigitte et moi, retirer pour la première fois, au distributeur automatique, les premiers euros, la monnaie commune!

N'oubliez pas l'impact du geste d'union Mitterrand-Kohl en septembre 1984 devant l'ossuaire de Douaumont. C'est dans cette atmosphère qu'elle obtient un jour, avec l'appui du président, de créer, adossé au programme Voltaire, ce qui deviendra le programme Brigitte Sauzay. Les deux sont bien connus des lycéens et de leurs professeurs. Et ce n'est pas facile, car il y a à l'époque comme aujourd'hui au Ministère comme au Rectorat, tout un parti pour dénoncer le projet comme élitiste et s'y opposer fermement, et d'autant plus que la gauche est au pouvoir... Il a fallu de la persévérance et le soutien du président.

La foi en l'Europe, comme toute foi, résistait à cette époque et elle résiste encore aujourd'hui, comme nous le voyons à travers les discours du président Macron, y compris le plus récent à la foire de Francfort $^{(3)}$, à la critique certainement justifiée de la réalité d'une union européenne technocratique et sans âme. Un souvenir personnel , si vous permettez: en 2005, j'enseignais à Sciences Po et je me souviens de mon arrivée le lundi matin, suivant le vote disant non à Maastricht: il régnait une sorte de désolation, une atmosphère au moins de sidération. Mais revenons à Brigitte.

Parler de l'Allemagne et des Allemands, c'est très prétentieux, bien sûr et, chacun avec son histoire, sa personnalité privilégie tel ou tel aspect. Qu'est-ce qui plaisait tant à Brigitte dans cet univers culturel?

Son premier contact avec la société allemande lui avait permis de découvrir un milieu très particulier, celui des aristocrates prussiens, milieu souvent francophone et francophile dont elle appréciait la culture et le raffinement, d'autant plus qu'il s'agissait d'un milieu laminé par la guerre. Elle avait ensuite, par le biais de la politique et de l'Université, noué des liens dans les milieux intellectuels et la gauche allemande, un peu à cause de la dimension écologique, où les Allemands étaient très en avance, et contestatrice, un peu seulement car Brigitte était un peu déroutée par les Verts. Je l'entends encore me dire «je suis sûre que tu serais verte jusqu'au bout des ongles si tu vivais en Allemagne!»

2 Jean-Michel Pouget, «L’union franco-allemande, 'épine dorsale' du 'grand organisme européen': Paul Heinrich Distelbarth (1879-1963) et le rêve d'une Europe organique», in: J.-M. Pouget (éd.), Conflit et coopération (note 1), p. 175-196.

3 Discours du 10 octobre 2017, http://www.elysee.fr/declarations/article/discours-du-president-de-larepublique-en-ouverture-de-la-foire-du-livre-de-francfort/ 
Non, ce qu'elle aimait dans la gauche allemande, c'était d'abord au plan intellectuel son interprétation de la philosophie des Lumières, sa façon de se situer dans le droit fil de la Réforme, de ne pas être antireligieuse contrairement à ce qui se passe en France. Elle appréciait précisément ce que Nietzsche reprochait aux Lumières allemandes, si j'ai bien lu Gilbert Merlio ${ }^{(4)}$. Elle trouvait très certainement que la social-démocratie allemande était supérieure à son équivalent français, ne serait-ce qu'à cause des rapports sociaux dans le monde du travail ou de la déclaration de Bad Godesberg.

Elle considérait que l'ironie, la légèreté, l'individualisme contestataire des Français gagnaient à être équilibrés par un goût du sentiment, du sérieux, du respect des règles plus marqué chez les Allemands. Je me souviens de conversations entre Rudolf von Thadden et elle sur le thème de la peur: les peurs françaises, peur de l'ennui, peur du ridicule, peur du déclin, s'opposaient aux peurs allemandes, peur du désordre, peur de ne pas comprendre; ensemble ils ambitionnaient de rédiger un dictionnaire des mots intraduisibles d'une langue vers l'autre.

Sur la situation des femmes, elle considérait au contraire que la société française était largement plus favorable et considérait comme un devoir de le faire savoir. Elle gardait un souvenir cuisant d'avoir été traitée de "Rabenmutter», de marâtre, dans un pays où le travail des femmes était encore suspect.

Elle avait aussi une vraie réflexion sur le nazisme et le dévoiement du désir de pureté qui, joint à la volonté de puissance, conduit Lucifer, le porte lumière en latin, le plus beau des anges, à incarner le Mal. C'est toute cette réflexion qui a donné lieu à la rédaction de son livre, journal comparé de l'année 1997, dans lequel elle commente les événements en croisant les perspectives allemande et française ${ }^{(5)}$.

Et c'est alors qu'intervient pour la troisième fois le rôle de la Chance dans la vie de Brigitte. La Fortune aux yeux bandés qui l'avait introduite à l'Élysée, qui lui avait fait rencontrer Rudolf von Thadden, lui offre une nouvelle opportunité: son livre vient d'avoir une excellente critique du président Richard von Weizsäcker qui la connaît depuis longtemps, quand elle est appelée au début de l'été par Gerhard Schröder, candidat du SPD aux élections de l'automne: il souffre auprès de la gauche allemande et française d'une image un peu trop libérale et d'une proximité revendiquée avec l'Angleterre. Afficher sa proximité avec l'interprète d'un président français, président socialiste, choisir une femme de surcroît, c'est améliorer son image et bénéficier de son soutien pendant la campagne: s'il est élu, elle aura à la Chancellerie un bureau et le poste officiel de conseillère pour les Affaires franco-allemandes. On connaît la suite: je suis allée à la Chancellerie à cette époque-là et j'ai vu un aspect de la façon dont Brigitte travaillait. Le chancelier préparant son discours pour une visite d'État, elle lui fournit des notes et lui conseille de vive voix de citer des vers, d'insérer de la poésie dans son texte pour séduire les Français et casser l'économisme qu'on lui reproche. Ce qui fut fait avec les résultats escomptés.

On pourrait s'étendre évidemment bien davantage sur l'action, l'influence, la vision de Brigitte Sauzay, mais ce n'est pas le lieu, on pourra, s'il est des curieux parmi vous,

4 Gilbert Merlio, «Le couple franco-allemand et la Figure du bon Européen chez Nietzsche», in: J.-M. Pouget (éd.), Conflit et coopération (note 1), p. 83-94.

5 Brigitte SAuzay, Retour à Berlin. Journal d'Allemagne, Paris, Plon, 1997. 
lire ses livres. On pourra aussi lire les belles pages que lui consacre Gerhard Schröder dans ses Mémoires ${ }^{(6)}$, et je vous y invite expressément. On trouvera je pense que tout ceci n'a pas pris une ride et que se plonger dans ces pages pour les germanistes distingués que vous êtes, c'est parler «les mots de la tribu», s’insérer de manière sympathique dans la cohorte de tous ceux qui, passeurs de culture, dans un combat toujours renouvelé et toujours nécessaire pour la Paix en Europe, méritent à mon sens, et j’inclus ici Anne-Marie Saint-Gille à qui est dédiée cette journée, oui ils méritent à mon sens, comme dirait Virgile, d’accéder aux champs élyséens.

6 Gerhard Schröder, Entscheidungen. Mein Leben in der Politik, Hambourg, Hoffmann und Campe, 2006. 